\title{
Research on Multi-mode Network Dance Movement Collection \& Evaluation System
}

\author{
Yiwen Zheng ${ }^{1}$, Chao Peng ${ }^{2}$, Yunhui Bai $^{3}$, Wenhong Zhao ${ }^{4}$ \\ ${ }^{1}$ department of Film and TV Technology, Zhejiang Vocational Academy of Art, Hangzhou, 310053, China \\ ${ }^{3}$ Department of Basic Teaching, Zhejiang Vocational Academy of Art, Hangzhou, 310053, China \\ ${ }^{2.4}$ College of Mechanical Engineering, Zhejiang University of Technology, Hangzhou, 310014, China
}

\begin{abstract}
At present, whether the students' dance movements are standard is usually evaluated by subjective methods in the dance teaching process. Therefore, there is often difference in subjective judgment and limitation to human eye observational ability caused by movement velocity. In this paper, the author designed and realized a dance movement scoring system based on gyroscope accelerometer and utilized the multi-node network technology for processing of accelerated speed and angle information collected at various limbs, so as to obtain real-time displacement coordinates and attitude angles. Upon the contrast between curves composed of sequences changing with the time and standard movement curve, the author obtained the overall similarity degree. The experiment has proved that this system is of relatively accurate collection for dance movements. Thus, it can faithfully reflect the movement conditions of limbs in dance movements and provide objective reference data for the evaluation.
\end{abstract}

Key words: gyroscope, multi-node network, displacement, attitude angle, similarity

\section{Preface}

Dance is limb movement with music rhythm. Its basic elements are movement posture, rhythm and expression. The stroking technique of dance has strict regulations in both space and time. It is very difficult to present this complicated three-dimensional spatial movement with simple, direct and understandable figures. Therefore, dance is always deemed as a kind of art which can only be unspeakable. For dance teachers and students in the dance major, it is of great importance for limb movement data in the process of learning, training and performance. This data is helpful for analyzing the presentation conditions of dance movements and skills. However, it is challenging for the issue of how to accurately measure this data. At present, students' dance movements and performances are directly observed and demonstrated by teachers in the teaching process of dance and then evaluated and guided through the method of subjective evaluation. This is taken as a kind of information feedback to help students to improve their movement performance and technical level. This evaluation method will always be influenced by many factors, such as age, hobby, emotion, time and speed of dance movement. Therefore, it is of certain subjectivity, lacking quantitative data foundation.

In the human motion capture and analysis system, there is a category of methods based on the 
computer visual principle ${ }^{[1]}$, which extracts and processes image sequences containing human motion by tracking a specific light spot on the target, so as to obtain human motion information. However, this system is very expensive. Moreover, it is relatively complex for follow-up processing of data. Another method is the recognition technology based on inertial sensor ${ }^{2[2-5]}$. At present, it has been widely applied in various fields, including national defense, sports competition and game \& animation making. In this paper, the movement sensor is used for real-time collection of limb information of dance movement. Changes in attitude angle and displacement track are obtained by processing the data collected, so as to realize the digitization of movement information. The deviation of dance movement relative to the standard demonstration movement can be judged through the data from the upper computer. Experiment has proved that this system can realize quantitative monitoring and attitude analysis of dance movement, providing objective indicator reference to subjective judgment. Moreover, it is characterized with high reliability, large range of measurement and low price.

\section{System Design}

Dance movement is a kind of mechanical motion compatible with the laws of mechanics as well as a kind of complicated and elaborate physiological motion. Analyzed from the perspective of exercise anatomy, three axes are arranged at human body: sagittal axis in the front and back direction, coronal axis in the left and right direction and vertical axis in the up and down direction. According to these axes, the human body is cut into three planes: sagittal plane, coronal plane and horizontal plane ${ }^{3[6]}$. Each dance movement is about exercise of the human body's motion link with joint as the supporting point, namely the movement of the motion link centering on the three basic axes on the three basic planes. It contains the following parameters: initial position, direction, acceleration, angle and displacement track. There are seven limb positions related to dance movement: hands, head, shoulder joint, waist, crotch, legs and feet. In the design on this system, various nodes of the sensor network are placed into these positions of human body for data collection and sampling.

The overall design plan of the system is shown in Fig. 1. It is mainly composed of senor module, USB-Wireless module and upper computer software. The working flow of the system is shown as follows: wear the sensor at key limb positions correlated with dance movement (such as forehead, left \& right big arms, left \& right small arms, left \& right big thighs, left \& right shanks, back of the hand, waist, chest, backs of left \& right feet and backs of left \& right hands) (for the convenience of wearing, the motion collection sensor is arranged inside of the bandage); in the process of dance movement, initialize the system through the upper computer software and control the data synchronously collected from various sensors; the processor embedded in the 
sensor will calculate once the attitude angle and the acceleration magnitude according to the frequency of $500 \mathrm{HZ}$; then, wireless launchers of various modules will send the information of attitude angle and acceleration magnitude to USB-Wireless module; the USB-Wireless module will send the data to the upper computer; at last, the data is subject to feature extraction by the upper computer software, so as to obtain the displacement-attitude curve and obtain evaluation results together with the data on standard dance movement according to RMSD algorithm.

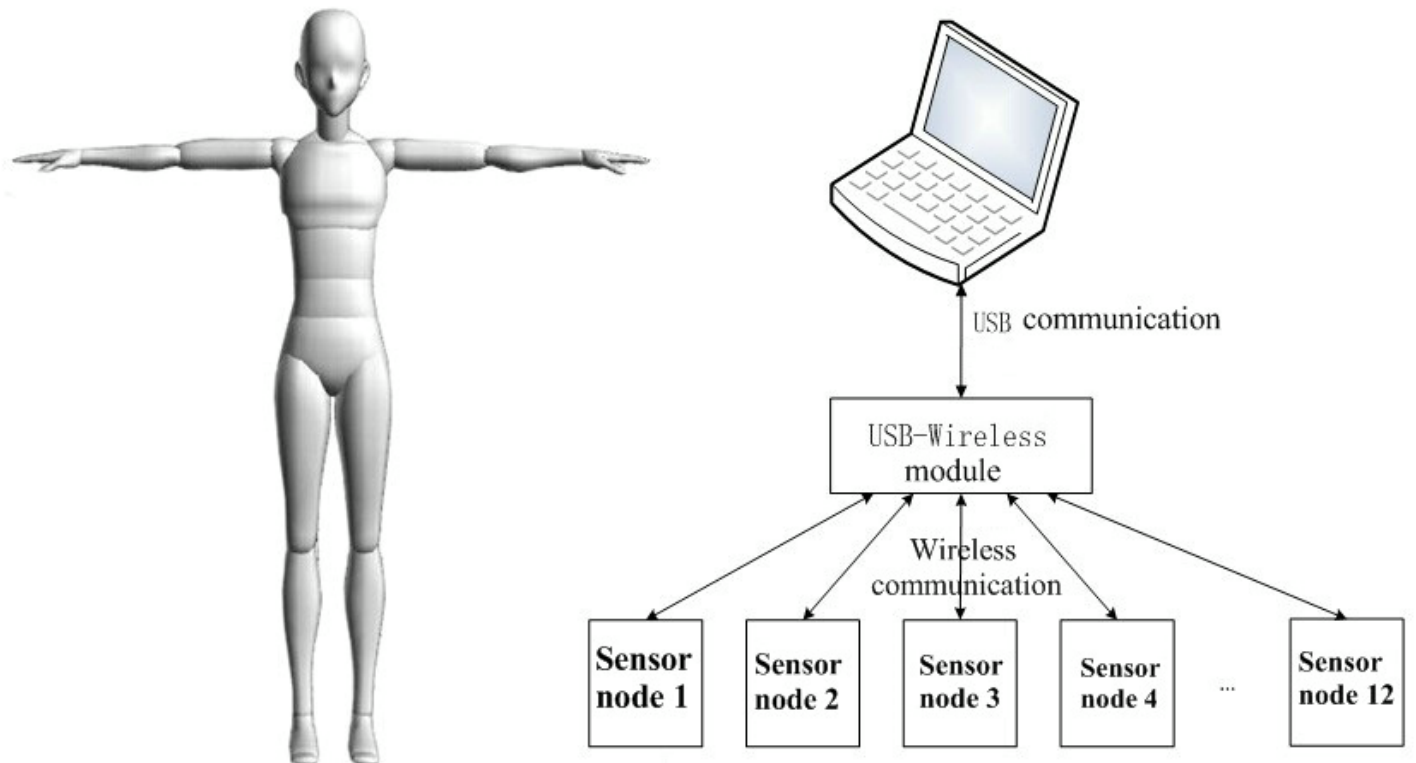

Fig. 1 General System Plan

\section{Composition of Hardware Structure}

Several sensor network nodes are adopted in this system. All nodes are mutually independent. They are composed of MPU6050 integrated 3-axis gyroscope and 3-axis MEMS accelerometer, magnetometer HMC588 and singlechip STM32. MPU6050 integrated 3-axis MEMS gyroscope and 3-axis MEMS accelerometer is of 16 ADCs, which can be used for accurate measurement of slow and fast movements. The range of measurement can reach $\pm 2 \sim \pm 16 \mathrm{~g}$ and $\pm 2 \sim \pm 2 \mathbf{0 0 0}^{\circ} / \mathrm{s}$. It has two IIC ports for auxiliary communication of IIC and magnetometer HMC588. The main IIC port is used for communication with the singlechip, real-time collection of data on 9 axes and complementation. The filter realizes the integration of data on 9 axes. This data is sent to the upper computer via XL24L01.

USB-Wireless module is the core of the entire system. This module is composed of processor STM32, USB chip FT245L and wireless receiving and dispatching chip XL24L01. Served as a connecting link between the preceding and the following, it is mainly used ordered 
communication between various sensor nodes and the upper computer ${ }^{4[7]}$. During a dance movement, we need to know the movement state information of various limbs at the same moment. Therefore, various modules must have favorable synchronism. USB-Wireless module sends synchronous orders in the form of broadcasting and then waits for answering signals from all sensor nodes. At last, it realizes synchronous collection of signals from all modules. After the completion of data collection, the data is orderly sent to USB-Wireless module.

\section{Calculation of Displacement and Attitude}

Dance movement evaluation in this system mainly refers to attitude and position of limbs. The process of dance movement can be regarded as the change process of the carrier coordinate system relative to the reference coordinate system in terms of position and attitude ${ }^{5[8]}$. The information of position and attitude can be obtained through the calculation of accelerometer, magnetometer and gyroscope ${ }^{6[9-10]} . \mathrm{X}, \mathrm{Y}$ and $\mathrm{Z}$ of the local northeastern coordinate system are selected as the reference coordinate system and $\mathrm{X}_{\mathrm{b}} \mathrm{Y}_{\mathrm{b}} \mathrm{Z}_{\mathrm{b}}$ as the carrier coordinate system. The collected dance movement attitude information is the obtained pitching angle $\theta$, course angle $\varphi$ and roll angel $\gamma \cdot \mathrm{X}_{\mathrm{b}} \mathrm{Y}_{\mathrm{b}} \mathrm{Z}_{\mathrm{b}}$ can be obtained by rotating $\varphi$ around $\mathrm{Z}, \theta$ around $\mathrm{Y}$ and $\gamma$ around $\mathrm{X}$.

1. Calculation of attitude angle

Gyroscope can be used for real-time measurement of angular velocity. The attitude quaternion ${ }^{7[11]}$ is subject to numerical integration through four-order Runge-Kutta method, so as to obtain real-time information of attitude angle. Accelerometer and magnetometer can also be used to measure attitude angle.

There are many solving methods of attitude angle, including Euler algorithm, direction cosine method and quaternion method. In this paper, the quaternion method is used to solve attitude angle due to its characteristics of reduced calculated amount, timeliness and singular point free. According to the relationship between attitude quaternion and angular velocity in Reference [11], it can be also expressed as the Formula (4-1):

Quaternion q is passed on according to the following equation:

$$
\begin{gathered}
\dot{q}=H_{b}(t) q(t) \\
{\left[\begin{array}{c}
\dot{q}_{0} \\
\dot{q}_{1} \\
\dot{q}_{2} \\
\dot{q}_{3}
\end{array}\right]=\frac{1}{2}\left[\begin{array}{cccc}
0 & -w_{\mathrm{bx}} & -w_{\mathrm{by}} & -w_{b z} \\
w_{b x} & 0 & w_{b z} & -w_{b y} \\
w_{b y} & -w_{b z} & 0 & w_{b x} \\
w_{b z} & w_{b y} & -w_{b x} & 0
\end{array}\right] \cdot\left[\begin{array}{l}
q_{0} \\
q_{1} \\
q_{2} \\
q_{3}
\end{array}\right]}
\end{gathered}
$$


Wherein: $w_{b x}, w_{b y}, w_{b z}$ is the angular velocity of $\mathrm{x}, \mathrm{y}$ and $\mathrm{z}$ measured by gyroscope. The four-order Runge-Kutta method is used for numerical integration. $\mathrm{T}$ is the renewal step length. The calculation process is shown as follows:

The final result is shown as follows:

$$
q(t+T)=q(t)+\frac{T}{6}\left[K_{1}+2 K_{2}+2 K_{3}+K_{4}\right]
$$

Obtain the quaternion parameter of the definition orientation, and then use the quaternion to obtain the equivalent direction cosine matrix; attitude angle can be calculated by the direction cosine matrix:

$$
\begin{aligned}
& \text { Course angle } \varphi=\arctan \frac{C_{12}}{C_{11}} \\
& \text { Roll angle } \gamma=\arctan \frac{C_{23}}{C_{33}}
\end{aligned}
$$

$$
\text { Pitching angle } \theta=-\arcsin C_{13}
$$

Gyroscope is of high reliability in terms of its value in a short period. Due to temperature variation, noise intervention, fixed measurement deviation of gyroscope, however, the accumulation of deviation will be caused. Therefore, accelerometer and magnetometer should be used to correct the deviation of gyroscope.

Under initial situation, the carrier coordinate system coincides with the northeastern coordinate system. According to the relationship of trigonometric function:

$$
\begin{gathered}
\theta=\arcsin \frac{g_{y}}{g \cos \theta} \\
\gamma=-\arcsin \frac{g_{x}}{g}
\end{gathered}
$$

Course angle is measured through magnetometer. According to Reference [12]:

$$
\varphi=\arctan \frac{M_{y} \cos \gamma-M_{z} \sin \gamma}{M_{x} \cos \theta+M_{y} \sin \theta \sin \gamma+M_{z} \cos \gamma \sin \theta}
$$

2. Integration of complementary filtering

It is of high reliability in terms of its value in a short period. Due to temperature variation, noise intervention, fixed measurement deviation of gyroscope, however, the rotation angle obtained by integral will accumulate deviation. However, accelerometer and magnetometer are free from such issue. Both sensors can complement each other's advantages. The attitude angle integrated by complementary filter is adopted.

Gyroscope is of good dynamic response. Its high frequency part is needed. For accelerometer, it is 
relatively sensitive to the function of human motion. The attitude angle obtained by taking its instant value is of relatively great deviation. Therefore, its low frequency part is used. The formula of complementary filter is shown as follows:

$$
\mathrm{f}(\mathrm{t})=\frac{\mathrm{k}}{s+k} m(t)+\frac{s}{s+k} n(t)
$$

Wherein: $\frac{\mathrm{k}}{s+k}$ can only pass through low frequency signals and $\frac{s}{s+k}$ through high frequency signals; $\mathrm{m}(\mathrm{t})$ is the attitude signal measured by accelerometer and magnetometer. $\mathrm{n}(\mathrm{t})$ is the attitude angle signal calculated by gyroscope. Selection of different k values under different conditions can make the system to have favorable performance.

When $f_{a}>f g$, reduce the value of $\mathrm{k}$; when $f_{a} \leq f g$, the value of $\mathrm{k}$ remains unchanged. $\mathrm{k}$ is a constant. $f_{a}=\sqrt{g_{x}^{2}+g_{x}^{2}+g_{z}^{2}}, \mathrm{~g}$ is acceleration of gravity.

3. Calculation of displacement

Accelerometer can be used to measure the displacement track of limbs, but the measured values of three axes of the accelerometer all include the component of acceleration of gravity, seriously disturbing the calculation of displacement. The carrier coordinate system projects the accelerated value into the reference coordinate system through the state-transition matrix. Therefore, the displacement must remove the component of acceleration of gravity on three axes of $\mathrm{X}, \mathrm{Y}$ and $\mathrm{Z}$.

$$
\left[\begin{array}{l}
a_{t x} \\
a_{t y} \\
a_{t z}
\end{array}\right]=C_{b}^{t}\left[\begin{array}{l}
a_{1 x} \\
a_{1 y} \\
a_{\mathrm{l} z}
\end{array}\right]-\left[\begin{array}{l}
0 \\
0 \\
g
\end{array}\right]
$$

Wherein: $\mathrm{Cb}$ is an orthogonal matrix, $C_{b}^{t}=C_{t}^{b^{T}}$

Obtain the velocity through integration of the accelerated speed at three axes X, Y and Z, and then obtain the displacement of $\mathrm{X}, \mathrm{Y}$ and $\mathrm{Z}$ through integration, so as to obtain the displacement coordinates of the three axes:

$$
V_{i}=V_{i-1}+\int_{0}^{t} a d t
$$

Wherein: $V_{i}$ is the current velocity; $V_{i-1}$ is the acceleration magnitude at the last moment; and $\mathrm{t}$ is the time interval of sampling.

$S_{i}=S_{i-1}+V_{i} t+\frac{1}{2} a t^{2}$

$S_{i}$ is the position of movement at the present moment; and $S_{i-1}$ is the position at the last moment. 


\section{Analysis on similarity}

Remake and record the motion information $\mathrm{B}$, use the upper computer software to make the correlation curve of displacement and attitude with the motion sequence of A and B; Fig. 2, Fig. 3 and Fig. 4 show the comparison of three attitude angles in Roll, Pitch and Yaw as well as displacement and track.
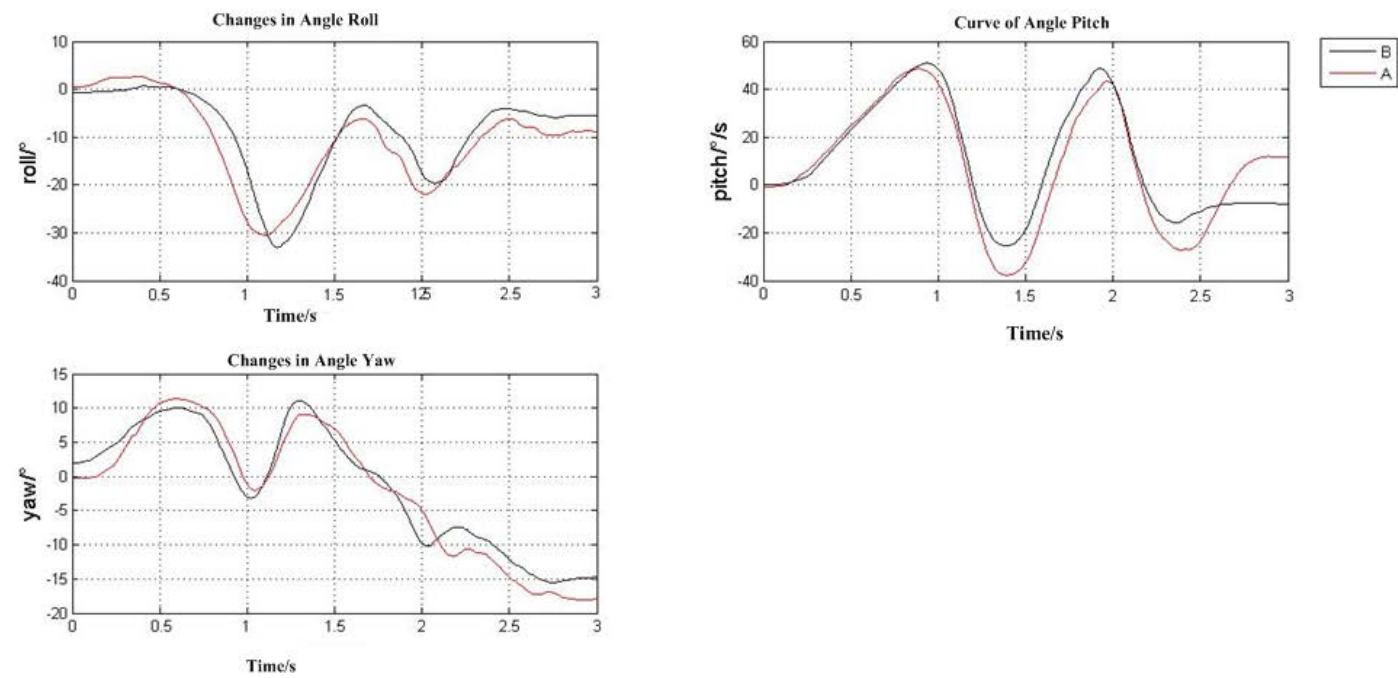

Fig. 2 Comparison of Attitude Angle

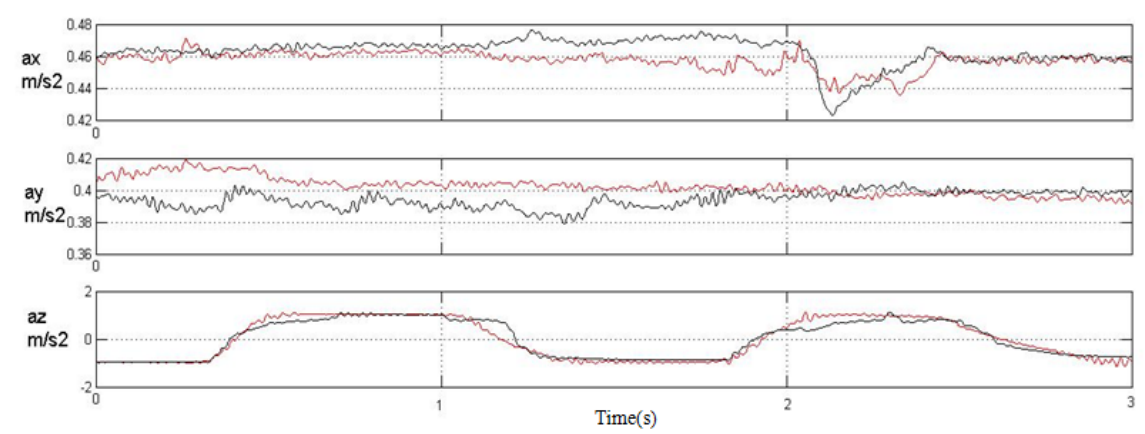

Fig. 3 Correlation Curve of Acceleration

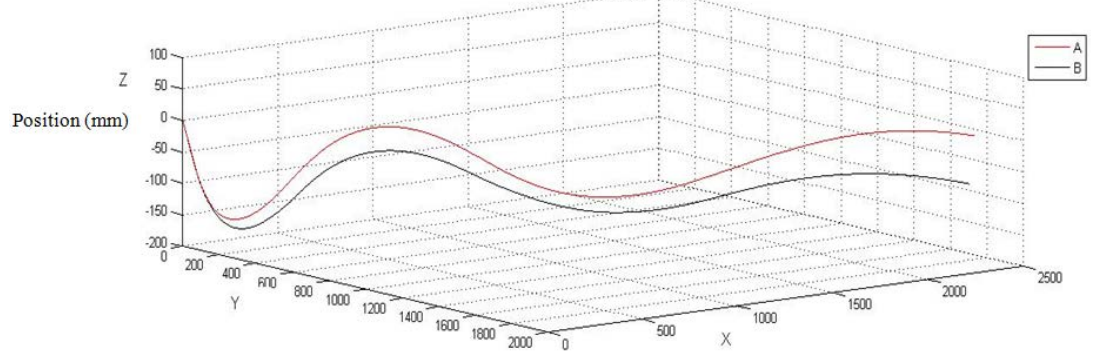

Fig. 4 Correlation Curve of Track

To obtain the similarity of movements A and B, the RMSD analysis method based on the motion curve similarity is adopted ${ }^{8[13-15]}$, namely the position and attitude coordinate sequence of two dance movements to be compared $\mathrm{S}=\left(s_{1}, s_{2}, \ldots s_{\mathrm{m}}\right), \mathrm{D}=\left(d_{1}, d_{2}, \ldots d_{n}\right) . \mathrm{S}$ stands for the sequence 
of dance movements. $d_{i}$ 和 $s_{i}$ respectively stand for coordinates of students' movements and standard movements at the moment $\mathrm{i}$ in the spatial coordinate system. Special displacement sequence is presented as $\left[\begin{array}{lll}d_{i x} & d_{i y} & d_{i z}\end{array}\right]^{T},\left[\begin{array}{lll}s_{i x} & s_{i y} & s_{i z}\end{array}\right]^{T}$. With the length $\mathrm{m}$ of sequence $\mathrm{S}$ as the standard, set $\mathrm{T}$ as the translation scale (unit: second), offset sequence $\mathrm{D}$ to $\mathrm{t}$ units; fill zero for any vacancy; at last, generate the sequence $\mathrm{D}=\left(d_{1}^{\prime}, d_{2}^{\prime}, \ldots d_{m}^{\prime}\right)$ with the same length as sequence $\mathrm{S}$.

$$
\operatorname{dis}_{\text {rmsd }}(D, S)=\left[\sqrt{\frac{1}{N} \sum_{i=1}^{N}\left\|d_{i}-s_{i}\right\|}\right]=\sqrt{\frac{1}{N} \sum_{i=1}^{N}\left(d_{i x}-w_{i x}\right)^{2}+\left(d_{i y}-w_{i y}\right)^{2}+\left(d_{i z}-w_{i z}\right)^{2}}
$$

Wherein: the value of $t$ is taken in sequence from the interval $[-500 \mathrm{~T}, 500 \mathrm{~T}]$, calculate $1000 \mathrm{~T}$ times with the above formula, take the minimum $d i S_{r m s d}$ as the optimal similarity, and then judge the subordinate range of $d i s_{r m s d}$. When $0<d i s_{r m s d}<\delta_{1}$, the performance is excellent; when $\delta_{1}<d i s_{r m s d}<\delta_{2}$, the performance is good; when $\delta_{2}<d i s_{r m s d}<\delta_{3}$, the performance is bad; when $\delta_{3}<d i s_{r m s d}<\delta_{4}$, the performance is very bad.

\section{Experimental data}

Fix the sensor onto key positions of limbs, perform the dance movement process, use the upper computer to record the data, take this data as the standard movement information $\mathrm{A}$, as shown in Fig. 1; it is a dispersed subsequence of this movement information, including the motion acceleration $a_{x}, a_{y}, a_{z}$ in three directions after the correction through direction cosine matrix; accelerate the integrated velocity at a time $v_{x}, v_{y}, v_{z}$ and accelerate the secondary integration to obtain displacement coordinates $\mathrm{x}, \mathrm{y}$ and $\mathrm{z}$ and measured attitude angles in real time: pitching angle $\theta$, course angle $\varphi$ and roll angle $\gamma$.

\begin{tabular}{|c|c|c|c|c|c|c|c|c|c|c|c|c|}
\hline numb & roll & pitch & yaw & $a x$ & ay & $\mathrm{az}$ & $\mathrm{vX}$ & $\mathrm{vy}$ & $\mathrm{Vz}$ & $\mathrm{x}$ & $y$ & $z$ \\
\hline 390 & -7.114 & 45.033 & 8.267 & 0.463 & 0.402 & 1.034 & 358.211 & 317.802 & -18.340 & 138.847 & 123.790 & -154.331 \\
\hline 391 & -7.740 & 45.566 & 7.910 & 0.463 & 404 & 1.025 & 359.136 & 318.607 & -16.279 & 139.564 & 124. 427 & -154.365 \\
\hline 392 & -8.443 & 46.055 & 7. 493 & 0.461 & 0.405 & 1.027 & 360.060 & 319.415 & -14.226 & 140.283 & 125.065 & -154. \\
\hline 393 & -9.251 & 46.478 & 7.015 & 0.465 & 0.404 & 1.038 & 0.986 & 320.222 & -12.164 & 141. 004 & 125.704 & -154.422 \\
\hline 394 & -10.096 & 46.824 & 6.493 & 0.464 & 0.404 & 1.024 & 1.915 & 321.029 & -10.107 & 141.727 & 126.346 & -154.445 \\
\hline 395 & -10.898 & 47.082 & 5.960 & 0.462 & 0.4 & 1.050 & 362.841 & .834 & -8.040 & 142. 452 & 126.989 & -154.463 \\
\hline 396 & -11.673 & 47.329 & 5.411 & 0.462 & 4 & & 64 & 39 & -5 . & 143.178 & 127.633 & -154.477 \\
\hline 397 & -12.530 & 47.576 & 4. 834 & 0.465 & 3 & & 91 & 47 & -3 & 143. & 279 & -154.487 \\
\hline 398 & -13.458 & 47.851 & 4. 224 & 0.463 & 0.4 & 1.0 & 19 & 4. 254 & -1.832 & 144. & 128.927 & -154.492 \\
\hline 399 & -14.403 & 48.148 & 3.631 & 0.462 & 0.403 & 1.0 & 44 & & 0.219 & 69 & 129.576 & -154.494 \\
\hline 400 & -15.370 & 48.406 & 65 & 0.463 & 0.4 & 25 & 70 & & 2. 277 & 103 & 227 & -154 \\
\hline 401 & -16.348 & 48. & 2. 532 & 0.462 & & & & & & & 880 & -154 \\
\hline 402 & -17.331 & 48. & 05 & & & & & & 6. & 577 & 534 & -154 \\
\hline 403 & -18.347 & 48.4 & & & & & & & 8.4 & & 190 & -154.459 \\
\hline 404 & -19.396 & 48.214 & 0.857 & 0.464 & 0.4 & 1. & 171 & & 10. & 558 & 847 & -154.440 \\
\hline 405 & -20.435 & 47.961 & 0.291 & 0.461 & 0.4 & 1.0 & .096 & & 12.615 & 149.801 & 506 & -154.417 \\
\hline 406 & -21.434 & 47.659 & -0.247 & 0.463 & 0.403 & 1. 031 & 373.021 & & 14.689 & 150.546 & 134.167 & -154.390 \\
\hline 407 & -22.396 & 47.285 & -0.786 & 0.462 & 0.404 & 1.035 & 373.946 & 331.521 & 16.758 & 151.293 & 134.829 & -154.358 \\
\hline 408 & -23.341 & 46.840 & -1.285 & 0.463 & 0.403 & 1.042 & 374.871 & 332.328 & 18.838 & 152.042 & 135.493 & -154.323 \\
\hline
\end{tabular}

\section{Conclusion}


The experimental result indicates that the multi-node network dance movement collection \& evaluation system can collect and measure changes in attitude angle and displacement track of limb movement in the process of dance movement, reflect the state of limbs, compare wave form changes according to the standard movements and evaluate the completion condition of movements. This system is of relatively high collection sensitivity, simple structure and low cost. The research on this system applied the electronic gyroscope technique into the process of dance teaching, providing objective parameter indicators for subjective evaluation of dance movements and providing theoretical basis and data reference for the optimization of students' dance movements.

\section{Acknowledgments}

This paper is the project of the Provincial Education Department (No.: Y201328493).

\section{References:}

[1] Yang Hui, Research on High Precision Pedestrian Navigation Algorithm based on MEMS Sensor [D], 2014.6;

[2] Gu Deming, Atlas of Exercise Anatomy [M], Peoples Sports Publishing House, 2013;

[3] Li Jianming, Design on USB Wireless Network System of nRF24LU1_[J], Microcontrollers \& Embedded Systems, 2009 (9): 35-39;

[4] Xu Bing, design and realization of human motion capture system based on MEMS sensor and ZigBee network [D], master's thesis of University of Electronic Science and Technology of China, 2013;

[5] Zhou Shucheng, Research on Three-dimensional Curve Matching Technique based on Multi-scale Analysis [J], Computer Applications and Software, 2006, (9): 51-60 